\title{
Editorial: Sustainable Production of Nutrient-Dense Foods
}

\author{
Autar K. Mattoo ${ }^{1 *}$, Urs Feller ${ }^{2}$ and Dan Roberts ${ }^{1}$ \\ ${ }^{1}$ United States Department of Agriculture, Agricultural Research Service, Sustainable Agricultural Systems Laboratory, \\ Beltsville, MD, United States, ${ }^{2}$ Institute of Plant Sciences and Oeschger Centre for Climate Change Research, University of \\ Bern, Bern, Switzerland
}

Keywords: food production, sustainable crops, nutritious foods, pulses, genetics, agriculture

\section{Editorial on the Research Topic}

\section{Sustainable Production of Nutrient-Dense Foods}

Feeding the world's growing population presents plant-based agriculture with enormous challenges as it is estimated that current agricultural production must increase 60 to 100 percent all else being unchanged (food waste levels, current trends in population growth and food consumption). The quantities and qualities of necessary food components, e.g., proteins, carbohydrates, lipids, and vitamins and minerals in plant products are highly relevant for human and animal consumption. These plant foods also contain a varied and limited range of bioactive compounds that have been associated with the prevention of chronic diseases including heart and circulation problems, cancer, diabetes, cataracts, and age-related functional decline.

Crop production cannot meet the nutritional demands of future populations simply by increasing land acreage devoted to agriculture. Competition for land use with urbanization, and poor land management will reduce suitable land available for agricultural production. It is also unlikely that productivity of current plant production systems can be increased by using the agricultural intensification methods of the past. Food production doubled worldwide over the past 35 years; largely due to the extensive use of synthetic fertilizer, pesticides, and irrigation. Feedstocks for synthetic fertilizer production are dwindling and water availability is already scarce in many regions of the world. In addition, agricultural intensification over the past decades has had negative environmental impacts, such as increased soil erosion and decreased soil fertility, pollution of ground water and eutrophication of rivers, lakes, and coastal ecosystems, and increased greenhouse gases. Overarching the problem of producing substantially more food is the increasingly erratic global climate. Global climate change is expected to lead to extreme weather events, increased temperatures, and increased concentrations of $\mathrm{CO}_{2}$ and ozone in the atmosphere. Increased temperatures will negatively impact yield of most crops in major production areas as yields decline drastically at temperatures above $30^{\circ} \mathrm{C}$. Flooding, drought, salinity stress, and higher ozone levels due to global climate change, as well as the associated development of new pest and pathogen problems, are predicted to be a drag on crop yields. Of equal concern are the negative impacts of elevated temperature on the quality of many crops.

Future production of nutrient dense food will require sustainable, climate-resilient, nextgeneration crop production systems, with decreased use of synthetic fertilizer and pesticides. Nextgeneration crop production systems will have to rely both on Big Data-driven precision agriculture, and biologically based technologies for increased soil health, pathogen and pest control, and control of soil erosion. New crop germplasm needs to be developed that has increased tolerance of biotic and abiotic stresses, and combinations of stresses; that offers higher yields but uses less water, fertilizer, pesticides, and other inputs; and has higher nutritional quality for the consumer. Crop germplasm development must also consider undesirable (e.g., phytic acid) or even toxic elements for humans and livestock (e.g., some heavy metals) found in certain edible plant tissues. Production 
of this germplasm will rely on state-of-the-art breeding strategies as well as advanced approaches using biotechnology, including genetic engineering.

We present here a collection of four articles, authored by experts in diverse disciplines, describing aspects of a Second Green Revolution where crop genetics are employed to develop germplasm to maintain the health of the human population and next-generation cropping systems are employed to maintain a healthy planet. The chapter by Delgado et al. discusses the use of Big Data from sensors within the field and aerial sensing systems, with Geographic Information Systems as the organizing principle, to increase agricultural system productivity and sustainability as well as enhance conservation of the environment through a precision agriculture approach. Taking this a step further, Delgado et al. introduce the concept of WebGIS to interconnect local, site-specific data generators called smart farms to create a regional and global view of agriculture that can support both the agricultural industry and policymakers in government. Ultimately, such a "system of systems approach" may enhance collaboration amongst scientists, policy makers, and farmers for speeding the development and adoption of technology by the agricultural community. The chapter by Roberts and Mattoo discusses the impacts of sustainable agricultural systems on crop nutrient content using a sustainable tomato production system as an example. This tomato production system uses a biologically based, cover crop approach to improve soil health and resilience to disease. Roberts and Mattoo also discuss the use of advanced approaches to develop tomato germplasm with improved qualities.

To meet the challenge of sustainably feeding the world population many different regional-specific approaches need to be developed and implemented. Countries that have not yet achieved food security face different challenges than those with sophisticated agricultural production systems. In such countries substantial yield increases may be achieved using improved materials (seed and fertilizer) commonly available in countries with sophisticated production systems, or through outreach providing informed advice. In countries that produce calorierich staple crops but insufficient micronutrient-rich non-staples such as vegetables, pulses, and animal products, micronutrient deficiencies will take hold and result in diseases concurrently afflicting two billion people. These situations call for adoption of new foodstuffs and/or for micronutrient fortification of the food supply including the use of specialized biofortified crop germplasm. The remaining two articles concern improving nutrition in human populations in sub-Sahara Africa. The first, by Gardner et al., discusses adoption of a new, nutritious crop, quinoa, in Malawi to supplement the use of the staple maize for human consumption. Using the Diffusion of Innovations framework the authors evaluated the adoption potential of quinoa and the importance of diffusion of information from extension programs to local farmers. The second, by Low et al., discusses breeding orange-fleshed type sweetpotato rich in pro-vitamin A and increasingly high in iron, for drought tolerance; management of sustainable productivity; and utilizing emerging cropping systems. They discuss the use of an accelerated sweetpotato breeding scheme to halve the time spent in the breeding cycle. They also present evidence that improved crop management can significantly increase sweetpotato yield.

\section{AUTHOR CONTRIBUTIONS}

All authors listed have made a substantial, direct and intellectual contribution to the work, and approved it for publication.

Conflict of Interest: The authors declare that the research was conducted in the absence of any commercial or financial relationships that could be construed as a potential conflict of interest.

Copyright (c) 2020 Mattoo, Feller and Roberts. This is an open-access article distributed under the terms of the Creative Commons Attribution License (CC BY). The use, distribution or reproduction in other forums is permitted, provided the original author(s) and the copyright owner(s) are credited and that the original publication in this journal is cited, in accordance with accepted academic practice. No use, distribution or reproduction is permitted which does not comply with these terms. 